\title{
Nonperturbative functional renormalization-group approach to the sine-Gordon model and the Lukyanov-Zamolodchikov conjecture
}

\author{
R. Daviet and N. Dupuis \\ Sorbonne Université, CNRS, Laboratoire de Physique Théorique de la Matière Condensée, LPTMC, F-75005 Paris, France
}

(Dated: December 5, 2018)

\begin{abstract}
We study the quantum sine-Gordon model within a nonperturbative functional renormalizationgroup approach (FRG). This approach is benchmarked by comparing our findings for the soliton and lightest breather (soliton-antisoliton bound state) masses to exact results. We then examine the validity of the Lukyanov-Zamolodchikov conjecture for the expectation value $\left\langle e^{\frac{i}{2} n \beta \varphi}\right\rangle$ of the exponential fields in the massive phase $(n$ is integer and $2 \pi / \beta$ denotes the periodicity of the potential in the sine-Gordon model). We find that the minimum of the relative and absolute disagreements between the FRG results and the conjecture is smaller than 0.01 .
\end{abstract}

Introduction. The quantum sine-Gordon model [1] describes many physical systems. In condensed matter it is widely used to understand the phase diagram and the low-energy properties of one-dimensional quantum fluids [2-4] and has applications that range from strongly correlated electron systems to cold atoms. In high-energy physics it is related to the massive Thirring model describing Dirac fermions with a self interaction [5]. The sine-Gordon model can also be viewed as a two-dimensional model of classical statistical mechanics. In particular it describes the Berezinskii-KosterlitzThouless (BKT) transition which occurs in the XY spin model and more generally in two-dimensional systems with a two-component order parameter with an $\mathrm{O}(2)$ symmetry [6-8].

The Hamiltonian of the quantum sine-Gordon model is defined by

$$
\hat{H}=\int d x\left\{\frac{1}{2} \hat{\Pi}^{2}+\frac{1}{2}\left(\frac{\partial \hat{\varphi}}{\partial x}\right)^{2}-u \cos (\beta \hat{\varphi})\right\}
$$

where $\hat{\Pi}$ and $\hat{\varphi}$ satisfy canonical commutation relations, $\left[\hat{\varphi}(x), \hat{\Pi}\left(x^{\prime}\right)\right]=i \delta\left(x-x^{\prime}\right)$. Regularization with a UV momentum cutoff $\Lambda$ is implied and $u / \Lambda^{2}, \beta>0$ are dimensionless parameters. The phase diagram consists of a gapless phase with massless (anti)soliton excitations for $\beta^{2} \geq 8 \pi$ (and $u \rightarrow 0$ ) and a gapped phase with massive (anti)soliton excitations for $\beta^{2} \leq 8 \pi$. The soliton and the antisoliton carry the topological charge $Q=1$ and -1 , respectively [9]. They attract for $\beta^{2} \leq 4 \pi$ and can form bound states, called breathers, with topological charge $Q=0$. The phase transition between the two phases is of BKT type [6-8].

The sine-Gordon model is one of the most studied integrable models; its spectrum, thermodynamics and scattering properties are well understood [10-14]. However not everything is known and many quantities can be obtained only from nonexact (e.g. perturbative) methods [2-4]. In particular in the massive phase the amplitude of the fluctuations about the mean value $\langle\hat{\varphi}\rangle=0$ is not known exactly. It has been conjectured by Lukyanov and Zamolodchikov that [15]

$$
\left\langle e^{i \sqrt{8 \pi} a \hat{\varphi}}\right\rangle=\left[\frac{\Gamma(1-K)}{\Gamma(K)} \frac{\pi u}{2(b \Lambda)^{2}}\right]^{\frac{a^{2}}{1-K}} \exp \left\{\int_{0}^{\infty} \frac{d t}{t}\left[\frac{\sinh ^{2}(2 a \sqrt{K} t)}{2 \sinh (K t) \sinh (t) \cosh [(1-K) t]}-2 a^{2} e^{-2 t}\right]\right\},
$$

where $|\Re(a)|<1 / 2 \sqrt{K}$ and $K=\beta^{2} / 8 \pi$ is the "Luttinger parameter" [4] (the massive phase corresponds to $K<1$ ). Equation (2) is exact for $a=\sqrt{K}, K=1 / 2$ and in the semiclassical limit $K \rightarrow 0$ [16]. Additional arguments supporting the conjecture were presented in [17, 18]. From the equivalence between the sine-Gordon model and the massive Thirring model Eq. (2) was shown to be correct to first order in $u[19,20]$. Further evidence of the correctness of (2), in particular for not too large values of $a$, was provided by a numerical study in a finite volume [21] and variational perturbation theory [22].

In this Letter, we examine the validity of the
Lukyanov-Zamolodchikov conjecture using a nonperturbative functional renormalization-group approach (FRG) [23, 24]. We go beyond previous FRG approaches [25-28] and, in order to benchmark our approach, first compute the mass $M_{\text {sol }}$ of the (anti)soliton as well as that $\left(M_{1}\right)$ of the lightest breather. We then turn to the computation of the expectation value $\left\langle e^{\frac{i}{2} n \beta \varphi}\right\rangle=$ $\left\langle e^{i n \sqrt{2 \pi K} \varphi}\right\rangle$ ( $n$ integer) of the exponential fields. We confirm the Lukyanov-Zamolodchikov conjecture with an accuracy, defined as the minimum of the relative and absolute disagreements between the FRG results and the 
conjecture, of 0.01 .

FRG approach. From now on we adopt the point of view of classical statistical mechanics (or Euclidean field theory) where the sine-Gordon model is defined by the partition function

$$
\mathcal{Z}[J]=\int \mathcal{D}[\varphi] e^{-\int d^{2} r\left\{\frac{1}{2}(\nabla \varphi)^{2}-u \cos (\beta \varphi)-J \varphi\right\}},
$$

with $\varphi(\mathbf{r})$ a classical field and $\mathbf{r}$ a two-dimensional coordinate. $J$ is an external source allowing us to obtain the expectation value $\phi(\mathbf{r})=\langle\varphi(\mathbf{r})\rangle=\delta \ln \mathcal{Z}[J] / \delta J(\mathbf{r})$ by functional derivation. Most physical quantities can be obtained from the free energy $-\ln \mathcal{Z}[J]$ or, equivalently, the effective action (or Gibbs free energy)

$$
\Gamma[\phi]=-\ln \mathcal{Z}[J]+\int d^{2} r J \phi
$$

defined as the Legendre transform of $\ln \mathcal{Z}[J]$.

We compute $\Gamma[\phi]$ using a Wilsonian nonperturbative FRG approach where fluctuation modes are progressively integrated out in the functional integral (3). This defines a scale-dependent effective action $\Gamma_{k}[\phi]$ which incorporates fluctuations with momenta between a (running) momentum scale $k$ and the UV scale $k_{\text {in }} \gg \Lambda$. The latter condition implies that the initial value $\Gamma_{k_{\text {in }}}[\phi]=S[\phi]$ coincides, as in mean-field theory, with the microscopic action defined by (3). The effective action of the sineGordon model, $\Gamma_{k=0}[\phi]$, is obtained when all fluctuations have been integrated out. The scale-dependent effective action satisfies an exact flow equation which cannot be solved exactly [29]. A common approximation scheme is the derivative expansion where

$$
\Gamma_{k}[\phi]=\int d^{2} r\left\{\frac{1}{2} Z_{k}(\phi)(\nabla \phi)^{2}+U_{k}(\phi)\right\}
$$

is truncated to second order in derivatives. This leads to coupled flow equations for the functions $Z_{k}(\phi)$ and

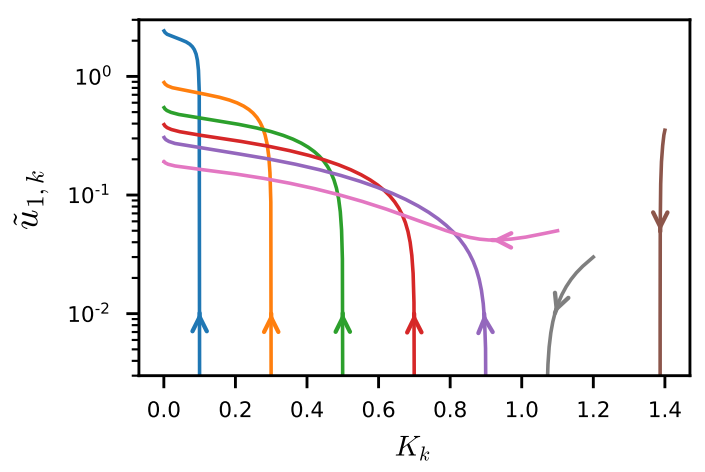

Figure 1. Flow diagram of the sine-Gordon model projected onto the plane $\left(K_{k}, \tilde{u}_{1, k}\right)$ where $\tilde{u}_{1, k}$ is the first harmonic of the potential $\tilde{U}_{k}(\phi)$ and $K_{k}$ the running Luttinger parameter. There is an attractive line of fixed points for $\tilde{u}_{1, k}=0$ and $K_{k}>1$ which terminates at the BKT point $\left(\tilde{u}_{1, k}=0, K_{k}=\right.$ 1).
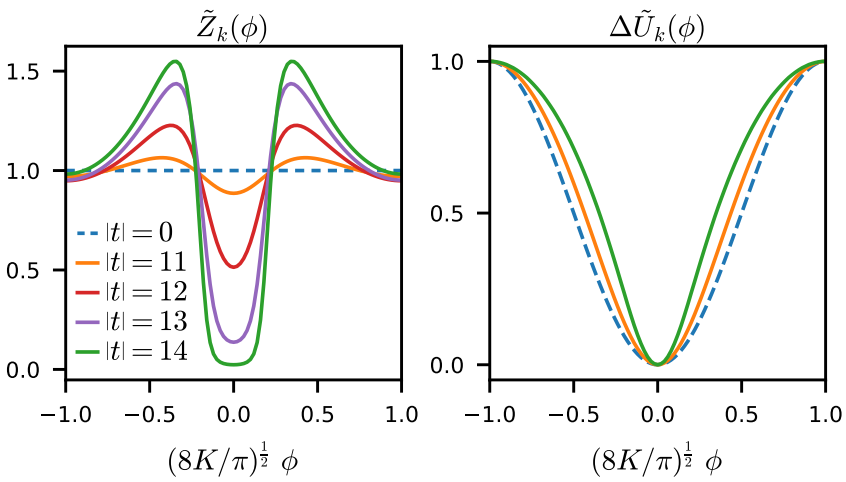

Figure 2. $\tilde{Z}_{k}(\phi)$ and $\tilde{U}_{k}(\phi)$ for various values of $t=$ $\ln \left(k / k_{\mathrm{in}}\right) . \Delta \tilde{U}_{k}(\phi)$ is given by $\tilde{U}_{k}(\phi)-\tilde{U}_{k}(0)$ normalized so that $\Delta \tilde{U}_{k}( \pm \sqrt{\pi / 8 K})=1$. In Figs. 2 and $3, \Lambda=1$ and $u / \Lambda^{2}=10^{-3}$.

$U_{k}(\phi)$, with initial conditions $Z_{k_{\text {in }}}(\phi)=1$ and $U_{k_{\text {in }}}(\phi)=$ $-u \cos (\beta \phi)$, which can be solved numerically. We refer to the Supplemental Material for more detail about the implementation of the FRG approach [30].

It is convenient to consider the dimensionless functions

$$
\tilde{Z}_{k}(\phi)=\frac{Z_{k}(\phi)}{Z_{k}}, \quad \tilde{U}_{k}(\phi)=\frac{U_{k}(\phi)}{Z_{k} k^{2}},
$$

where $Z_{k}=\left\langle Z_{k}(\phi)\right\rangle_{\phi}$ is obtained by averaging $Z_{k}(\phi)$ on ]$-\beta / \pi, \beta / \pi]$ [31]. The flow diagram, projected onto the plane $\left(K_{k}, \tilde{u}_{1, k}\right)$ is shown in Fig. 1 . Here $\tilde{u}_{1, k}$ is the first harmonic of the potential $\tilde{U}_{k}(\phi)=-\sum_{n=0}^{\infty} \tilde{u}_{n, k} \cos (n \beta \phi)$ and $K_{k}=K / Z_{k}$ can be interpreted as a "running" Luttinger parameter. In the massive phase, the flow runs into fixed points characterized by functions $\tilde{Z}^{*}(\phi)$ and $\tilde{U}^{*}(\phi)$ which depend on the parameters $u$ and $K$ (Fig. 2). While $\tilde{U}^{*}(\phi)$ slightly deviates from the cosine form of the initial potential $\tilde{U}_{k_{\text {in }}}(\phi)=-\left(u / k_{\text {in }}^{2}\right) \cos (\beta \phi)$, we see that $\tilde{Z}^{*}(\phi)$ acquires a strong dependence on $\phi . Z_{k}$ diverges as $k^{-2}$ and the running Luttinger parameter $K_{k} \sim k^{2}$ vanishes for $k \rightarrow 0$.

Benchmarking: soliton and breather masses. The smallest excitation gap $M$ of the quantum sine-Gordon model corresponds to the inverse correlation length of the two-dimensional classical model (3),

$$
M^{2}=\lim _{k \rightarrow 0} \frac{U_{k}^{\prime \prime}(0)}{Z_{k}(0)}=\lim _{k \rightarrow 0} k^{2} \frac{\tilde{U}_{k}^{\prime \prime}(0)}{\tilde{Z}_{k}(0)} .
$$

Since $\tilde{U}_{k}^{\prime \prime}(0)$ converges to a finite value -this property is preserved even if one retains only the first harmonics of $\tilde{U}_{k}(\phi)-\tilde{Z}_{k}(0)$ must vanish as $k^{2}$ for $M$ to take a nonzero value in the massive phase. $\tilde{Z}_{k}(\phi)$ being a normalized function, $\left\langle\tilde{Z}_{k}(\phi)\right\rangle_{\phi}=1$, this is possible only if $\tilde{Z}_{k}(\phi)$ strongly varies with $\phi$. Thus only a functional approach where the coefficient of $(\boldsymbol{\nabla} \phi)^{2}$ in the effective action is a function $Z_{k}(\phi)$, and not a mere $\phi$-independent number, can predict the mass of the lowest excitation. Numeri- 


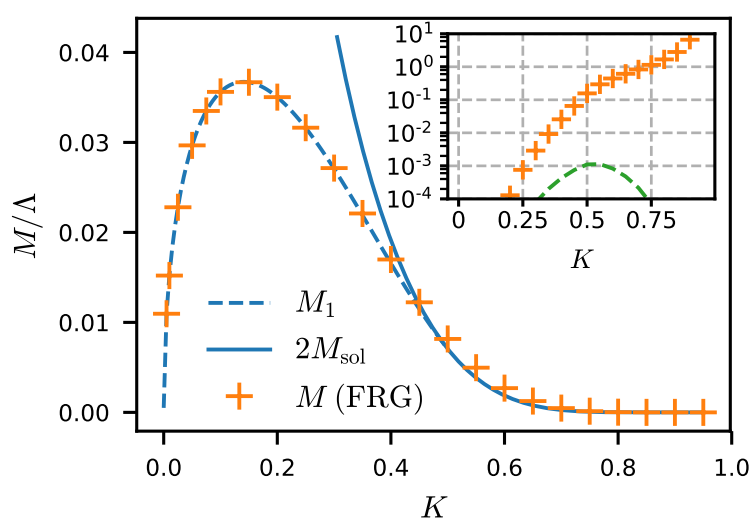

Figure 3. Mass $M$ of the lowest excitation as obtained from the FRG approach. The solid and dashed lines show the exact values of $2 M_{\text {sol }}$ and $M_{1}$ (the latter being defined only for $K \leq 1 / 2)$ [Eqs. $(8,9)$ ]. The inset shows the relative (crosses) and absolute (dashed line) errors of the FRG result.

cally we observe a rapid convergence of $k^{2} \tilde{U}_{k}^{\prime \prime}(0) / \tilde{Z}_{k}(0)$ when $k \rightarrow 0$ in agreement with a previous study [27].

Only excitations that are in the same topological sector as the ground state, namely $Q=0$, contribute to the mass $M$ [1]. The lowest excitation in this sector is a soliton-antisoliton pair with mass

$$
2 M_{\mathrm{sol}}=b \Lambda \frac{4 \Gamma\left(\frac{K}{2-2 K}\right)}{\sqrt{\pi} \Gamma\left(\frac{1}{2-2 K}\right)}\left[\frac{\Gamma(1-K)}{\Gamma(K)} \frac{\pi u}{2(b \Lambda)^{2}}\right]^{\frac{1}{2-2 K}},
$$

when $1 / 2 \leq K \leq 1\left(M_{\text {sol }}\right.$ is the mass of a single (anti)soliton) and a breather with mass

$$
M_{1}=2 M_{\mathrm{sol}} \sin \left(\frac{\pi}{2} \frac{K}{1-K}\right),
$$

when $0 \leq K<1 / 2[32]$. Here $b$ is a scale parameter which depends on the precise implementation of the UV cutoff $\Lambda$ in Eq. (1). Figure 3 shows the value of $M$ obtained from FRG (we refer to the Supplemental Material for a discussion of the implementation of the UV cutoff $\Lambda$ and the determination of the scale factor $b$ ). For $0 \leq K \leq 0.4$ our result for the breather mass $M \equiv M_{1}$ deviates from the exact value by at most $2 \%$. The agreement becomes nearly perfect for $K \ll 0.4$, which is due to the fact that the initial value $\Gamma_{k_{\text {in }}}^{(2)}(\mathbf{q}, \phi=0)=\mathbf{q}^{2}+8 \pi K u$ gives the exact breather mass $M_{1, \mathrm{cl}}=\sqrt{8 \pi K u}$ in the semiclassical limit $K \rightarrow 0$ [30]. For $0.4 \leq K \leq 1$ the agreement between $M$ and the exact value $2 M_{\text {sol }}$ is not as good and varies from $\sim 2 \%$ for $K \simeq 0.4$ to more than $100 \%$ for $K$ near 1 . Note however that $M$ goes to zero when $K \rightarrow 1$ and the absolute error remains below $10^{-3}$ for all values of $K$ (see the inset in Fig. 3). In the immediate vicinity of $K=1$, the behavior of the mass $M$ differs from $2 M_{\text {sol }}[$ Eq. (8)] and one recovers the standard BKT scaling characterized by an essential singularity of the correlation length [8].

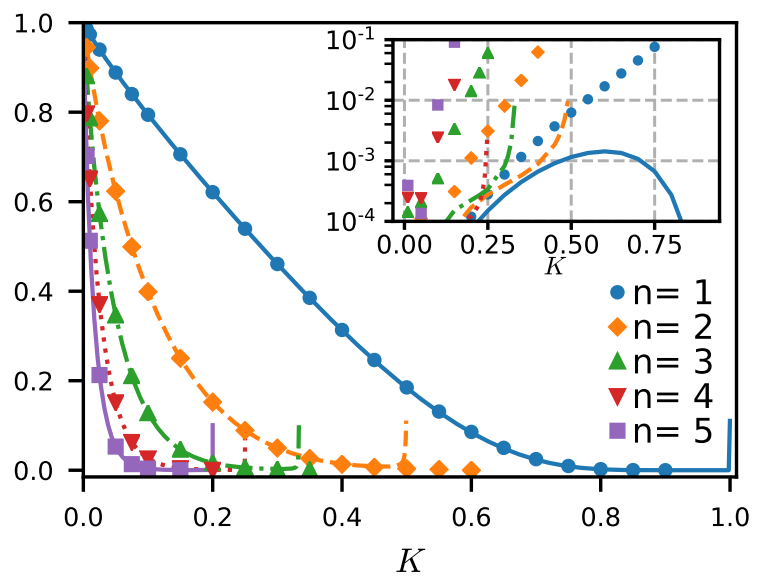

Figure 4. Expectation value $\left\langle e^{i n \sqrt{2 \pi K} \varphi}\right\rangle$ as obtained from FRG (symbols) vs the Lukyanov-Zamolodchikov conjecture (2) valid for $K<1 / n$ (lines). The inset shows the relative (symbols) and absolute (lines) disagreements between the FRG results and the conjecture, respectively $\epsilon_{\text {rel }}$ and $\epsilon_{\text {abs }}$. $\left(\Lambda=1\right.$ and $u / \Lambda^{2}=10^{-4}$.)

The Lukyanov-Zamolodchikov conjecture. To obtain the expectation value of the exponential fields, we consider the partition function (3) in the presence of an external source term $\int d^{2} r\left(h^{*} e^{i \sqrt{8 \pi} a \varphi}+\right.$ c.c. $)$ so that $\left\langle e^{i \sqrt{8 \pi} a \varphi(\mathbf{r})}\right\rangle$ can be obtained from $\ln \mathcal{Z}_{k}\left[J, h^{*}, h\right]$ by functional differentiation wrt $h^{*}(\mathbf{r})$. To second order of the derivative expansion the effective action now reads

$$
\begin{aligned}
\Gamma_{k}\left[\phi, h^{*}, h\right]= & \int d^{2} r\left\{\frac{1}{2} Z_{k}\left(\phi, h^{*}, h\right)(\nabla \phi)^{2}\right. \\
& \left.+U_{k}\left(\phi, h^{*}, h\right)\right\}
\end{aligned}
$$

and

$$
\left\langle e^{i \sqrt{8 \pi} a \varphi(\mathbf{r})}\right\rangle=-\left.\frac{\partial U_{k}\left(\phi=0, h^{*}, h\right)}{\partial h^{*}}\right|_{h^{*}=h=0} .
$$

From the flow equation of $\Gamma_{k}\left[\phi, h^{*}, h\right]$ we obtain two coupled equations for $\left.U_{k}^{(10)}(\phi) \equiv \partial_{h^{*}} U_{k}\left(\phi, h^{*}, h\right)\right|_{h^{*}=h=0}$ and $\left.Z_{k}^{(10)}(\phi) \equiv \partial_{h^{*}} Z_{k}\left(\phi, h^{*}, h\right)\right|_{h^{*}=h=0}$ with initial conditions $U_{k_{\text {in }}}^{(10)}(\phi)=-e^{i \sqrt{8 \pi} a \phi}$ and $Z_{k_{\text {in }}}^{(10)}(\phi)=0$ [30].

We have computed the expectation value of the exponential fields $e^{i n \sqrt{2 \pi K} \varphi}$ ( $n$ integer). These are the natural fields to consider in the sine-Gordon model. For instance, in one-dimensional quantum fluids, they arise from products of single-particle fields. The FRG results for $1 \leq n \leq 5$ are shown in Fig. 4 .

For $n=1$ we find an excellent agreement between the FRG results and the conjecture, with a difference $\epsilon_{\text {abs }}$ well below 0.01 for all values of $K$. The relative disagreement $\epsilon_{\text {rel }}$ is small for $K<0.5$ but increases for larger values of $K$ and becomes of order of $100 \%$ for $K$ near 1. For these values of $K$ however, the expectation value $\left\langle e^{i \sqrt{2 \pi K} \varphi}\right\rangle$ is very small and what matters is $\epsilon_{\mathrm{abs}}$. 
Note that the Lukyanov-Zamolodchikov conjecture breaks down in the vicinity of $K=1 / n$ since the expectation value $\left\langle e^{i n \sqrt{2 \pi K} \varphi}\right\rangle$ given by Eq. (2) diverges when $K \rightarrow 1 / n$ [33]. This explains the steep upturn near $K=1 / n$ of the lines showing the conjecture in Fig. 4. Decreasing the value of $u / \Lambda^{2}$ confines the upturn more and more to the vicinity of $1 / n$.

For $n \geq 2, \epsilon_{\text {rel }}$ behaves similarly to the case $n=1$ but $\epsilon_{\mathrm{abs}}$ is also a monotonously increasing function of $K$ (see the inset in Fig. 4). $\epsilon_{\text {abs }}$ remains nevertheless below 0.01 up to values of $K$ very close to $1 / n$; for $u / \Lambda^{2}=10^{-4}$ this is the case for $K=0.49$ (and $n=2$ ), $K=0.33$ $(n=3)$ and $K=0.248(n=4)$. Moreover $\epsilon_{\text {rel }}$ decreases when $u / \Lambda^{2}$ is reduced (which extends the domain of validity of the conjecture to higher values of $K$, i.e. to values of $K$ closer to $1 / n)$. For instance, for $n=2$ and $K=0.49$, we find $\epsilon_{\text {rel }}=78 / 72 / 66 \%$ (while $\epsilon_{\text {abs }}=$ $0.097 / 0.0097 / 0.00098)$ for $u / \Lambda^{2}=10^{-3} / 10^{-4} / 10^{-5}$. We therefore ascribe the apparent disagreement between the FRG results and the conjecture near $K=1 / n$ to the breakdown of the latter when $K \rightarrow 1 / n$. In fact the change of concavity in the curves showing $\epsilon_{\text {abs }}$ in the inset of Fig. 4 suggests that the conjecture might deviate from the correct result well before $K=1 / n$ (e.g. $K \sim 0.4$ for $n=2$ and $\left.u / \Lambda^{2}=10^{-4}\right)$. A conservative estimate is that the FRG reproduces Eq. (2), in the domain of validity of the conjecture, to an accuracy (defined as the minimum of $\epsilon_{\mathrm{abs}}$ and $\epsilon_{\mathrm{rel}}$ ) better than 0.01 .

Conclusion. Contrary to the perturbative RG [23, 34, 35 , which correctly predicts the phase diagram of the quantum sine-Gordon model but fails to describe the massive phase, the nonperturbative FRG allows us to continue the flow into the strong-coupling regime and thus compute the low-energy properties of the massive phase. The fact that FRG captures genuinely nonperturbative topological excitations, namely (anti)solitons and breathers, proves its efficiency and is reminiscent of its ability to describe most universal properties of the BKT transition in the linear $\mathrm{O}(2)$ model [36-38] for which it is widely admitted that topological defects (vortices) play a crucial role.

The FRG result for the expectation value $\left\langle e^{i n \sqrt{2 \pi K} \varphi}\right\rangle$ of the exponential fields is in very good agreement with the conjecture proposed by Lukyanov and Zamolodchikov [15]. The minimum of the relative and absolute disagreements is smaller than 0.01 for all values of $n$ except in the immediate vicinity of $K=1 / n$ where the conjecture breaks down. This undoubtedly provides us with a very strong support of the Lukyanov-Zamolodchikov conjecture. We also stress that FRG allows one to obtain $\left\langle e^{i n \sqrt{2 \pi K} \varphi}\right\rangle$ for all values of $K$ whereas the conjecture is limited to the range $K<1 / n$.

Finally we would like to point out that the nonperturbative FRG approach presented in this Letter opens up the possibility to study various nonintegrable exten- sions of the quantum sine-Gordon model where both perturbative RG and exacts methods are inoperative in the strong-coupling phase.

Acknowledgment. We thank P. Azaria for enlightening discussions and a critical reading of the manuscript.

[1] For an introduction to the quantum sine-Gordon model see R. Rajaraman, Solitons and instantons (North-Holland, Amsterdam, 1989).

[2] A. O. Gogolin, A. A. Nersesyan, and A. M. Tsvelik, Bosonization and strongly correlated systems (Cambridge University Press, 1998).

[3] Alexei M. Tsvelik, Quantum field theory in condensed matter physics, 2nd ed. (Cambridge University Press, 2007).

[4] T. Giamarchi, Quantum physics in one dimension (Oxford University Press, Oxford, 2004).

[5] Sidney Coleman, "Quantum sine-Gordon equation as the massive Thirring model," Phys. Rev. D 11, 2088-2097 (1975).

[6] V. L. Berezinskii, "Destruction of long-range order in onedimensional and two-dimensional systems having a continuous symmetry group i. classical systems," Sov. Phys. JETP 32, 493 (1971); "Destruction of long-range order in one-dimensional and two-dimensional systems possessing a continuous symmetry group. ii. quantum systems," Sov. Phys. JETP 34, 610 (1972).

[7] J. M. Kosterlitz and D. J. Thouless, "Ordering, metastability and phase transitions in two-dimensional systems," J. of Phys. C 6, 1181 (1973).

[8] J. M. Kosterlitz and D. J. Thouless, "The critical properties of the two-dimensional XY model," J. Phys. C 7, 1046 (1974).

[9] Classically the topological charge of a static field configuration is defined by $Q=(\beta / 2 \pi) \int_{-\infty}^{\infty} d x \partial_{x} \varphi=$ $(\beta / 2 \pi)[\varphi(\infty)-\varphi(-\infty)]$. In the quantum theory $Q$ becomes a topological quantum number [1].

[10] L. D. Faddeev and V. E. Korepin, "Quantum theory of solitons," Phys. Rep. 42, 1-87 (1978).

[11] E. K. Sklyanin, L. A. Takhtajan, and L. D. Faddeev, "The Quantum Inverse Problem Method. 1," Theor. Math. Phys. 40, 688-706 (1980), [Teor. Mat. Fiz.40,194(1979)].

[12] A. B. Zamolodchikov, "Exact two-particle S-matrix of quantum sine-Gordon solitons," Commun. Math. Phys. 55, 183-186 (1977).

[13] A. B. Zamolodchikov and Al. B. Zamolodchikov, "Factorized S-matrices in two dimensions as the exact solutions of certain relativistic quantum field theory models," Ann. Phys. 120, 253-291 (1979).

[14] F. A. Smirnov, Form Factors in Completely Integrable Models of Quantum Field Theory, Advanced series in mathematical physics, v. 14 (World Scientific, 1992).

[15] S. Lukyanov and A. B. Zamolodchikov, "Exact expectation values of local fields in the quantum sine-Gordon model," Nucl. Phys. B 493, 571 - 587 (1997).

[16] For $a=\sqrt{K}$ the expectation value $\left\langle e^{i \sqrt{8 \pi} a \varphi}\right\rangle$ is simply related to the free energy which is exactly known. For $K=1 / 2$ the exact value is deduced from the mapping onto the massless Thirring model [15]. 
[17] V. Fateev, S. Lukyanov, A. B. Zamolodchikov, and Al. B. Zamolodchikov, "Expectation values of boundary fields in the boundary sine-Gordon model," Phys. Lett. B 406, 8388 (1997).

[18] V. Fateev, S. Lukyanov, A. B. Zamolodchikov, and Al. B. Zamolodchikov, "Expectation values of local fields in the Bullough-Dodd model and integrable perturbed conformal field theories," Nucl. Phys. B 516, 652-674 (1998).

[19] R. H. Poghossian, "Perturbation theory in angular quantization approach and the expectation values of exponential fields in sine-Gordon model," Nucl. Phys. B 570, 506$522(2000)$.

[20] V. V. Mkhitaryan, R. H. Poghossian, and T. A. Sedrakyan, "Perturbation theory in the radial quantization approach and the expectation values of exponential fields in the sine-Gordon model," J. Phys. A 33, 3335-3346 (2000).

[21] Z. Bajnok, L. Palla, G. Takács, and F. Wágner, "The $k$-folded sine-Gordon model in finite volume," Nucl. Phys. B 587, 585-618 (2000).

[22] W.-F. Lu, "Sine-Gordon expectation values of exponential fields with variational perturbation theory," Phys. Lett. B 602, 261-268 (2004).

[23] The perturbative renormalization group is a popular method to study the sine-Gordon model [2-4]. In the massive phase however, perturbative scaling equations lead to an unphysical divergence of the coupling constant $u$. The phase diagram can be determined but the understanding of the physical properties of the massive phase is out of reach of the perturbative approach.

[24] Nonperturbative flow equations have been obtained from continuous unitary transformations but the determination of the the spectrum (soliton and breather's masses) and the validity of the Lukyanov-Zamolodchikov conjecture have not been addressed. See S. Kehrein, "Flow Equation Solution for the Weak- to Strong-Coupling Crossover in the Sine-Gordon Model," Phys. Rev. Lett. 83, 49144917 (1999); "Flow equation approach to the sine-Gordon model," Nucl. Phys. B 592, 512 - 562 (2001).

[25] S. Nagy, I. Nándori, J. Polonyi, and K. Sailer, "Functional Renormalization Group Approach to the SineGordon Model," Phys. Rev. Lett. 102, 241603 (2009).

[26] V. Pangon, "Structure of the broken phase of the sineGordon model using functional renormalisation," Int. J. Mod. Phys. A 27, 1250014 (2012).

[27] V. Pangon, "Generating the mass gap of the sine-Gordon model," (2011), arXiv:1111.6425.

[28] V. Bacsó, N. Defenu, A. Trombettoni, and I. Nándori, "c-function and central charge of the sine-Gordon model from the non-perturbative renormalization group flow ," Nucl. Phys. B 901, $444-460$ (2015).

[29] For reviews on the nonperturbative functional renormalization group, see J. Berges, N. Tetradis, and C. Wetterich, "Non-perturbative renormalization flow in quantum field theory and statistical physics," Phys. Rep. 363, 223 (2002); B. Delamotte, "An Introduction to the Nonperturbative Renormalization Group," in Renormalization Group and Effective Field Theory Approaches to ManyBody Systems, Lecture Notes in Physics, Vol. 852, edited by A. Schwenk and J. Polonyi (Springer Berlin Heidelberg, 2012) pp. 49-132; P. Kopietz, L. Bartosch, and F. Schütz, Introduction to the Functional Renormalization Group (Springer, Berlin, 2010).

[30] See Supplemental Material.
[31] Note that we do not rescale the field in order to preserve the $2 \pi / \beta$ periodicity of $Z_{k}(\phi)$ and $U_{k}(\phi)$.

[32] Al. B. Zamolodchikov, "Mass scale in the sine-Gordon model and its reductions," Int. J. Mod. Phys. A 10, 11251150 (1995).

[33] Near $K=1$ and $u=0$ the behavior of the system is controlled by the BKT fixed point, which explains why the conjecture cannot be valid arbitrary close to $K=1$.

[34] P. B. Wiegmann, "One-dimensional Fermi system and plane XY model," J. Phys. C. 11, 1583-1598 (1978).

[35] D. J. Amit, Y. Y. Goldschmidt, and S. Grinstein, "Renormalisation group analysis of the phase transition in the 2D Coulomb gas, Sine-Gordon theory and XY-model," J. Phys. A 13, 585 (1980).

[36] M. Gräter and C. Wetterich, "Kosterlitz-Thouless Phase Transition in the Two Dimensional Linear $\sigma$ Model," Phys. Rev. Lett. 75, 378-381 (1995).

[37] G. v. Gersdorff and C. Wetterich, "Nonperturbative renormalization flow and essential scaling for the Kosterlitz-Thouless transition," Phys. Rev. B 64, 054513 (2001).

[38] P. Jakubczyk, N. Dupuis, and B. Delamotte, "Reexamination of the nonperturbative renormalization-group approach to the Kosterlitz-Thouless transition," Phys. Rev. E 90, 062105 (2014). 


\section{Supplemental Material}

We consider the sine-Gordon model defined by the Euclidean action

$$
S[\varphi]=\int d^{2} r\left\{\frac{1}{2}(\nabla \varphi)^{2}-u \cos (\beta \varphi)\right\},
$$

where regularization with a UV momentum cutoff $\Lambda$ is implied and $u / \Lambda^{2}, \beta>0$ are dimensionless parameters. In the following, instead of the parameter $\beta$, we will often use the Luttinger parameter $K=\beta^{2} / 8 \pi$.

\section{EXACT FLOW EQUATION}

To implement the functional renormalization-group (FRG) approach we add to the action the infrared regulator term

$$
\Delta S_{k}[\varphi]=\frac{1}{2} \sum_{\mathbf{q}} \varphi(-\mathbf{q}) R_{k}(\mathbf{q}) \varphi(\mathbf{q})
$$

such that fluctuations are smoothly taken into account as $k$ is lowered from the microscopic scale $k_{\text {in }}$ down to 0 [1-3]. The regulator function in (2) is defined by

$$
R_{k}(\mathbf{q})=Z_{k} \mathbf{q}^{2} r\left(\frac{\mathbf{q}^{2}}{k^{2}}\right),
$$

where the function $r(y)$ satisfies $r(0)=\infty$ and $r(\infty)=0$, and $Z_{k}$ is a field renormalization factor defined below. Thus $\Delta S_{k}$ suppresses fluctuations with momenta $|\mathbf{q}| \ll k$ but leaves unaffected those with $|\mathbf{q}| \gg k$. Various choices for $r(y)$ are discussed below.

The partition function

$$
\mathcal{Z}_{k}[J]=\int \mathcal{D}[\varphi] e^{-S[\varphi]-\Delta S_{k}[\varphi]+\int d^{2} r J \varphi}
$$

is $k$ dependent. The scale-dependent effective action

$$
\Gamma_{k}[\phi]=-\ln \mathcal{Z}_{k}[J]+\int d^{2} r J \phi-\Delta S_{k}[\phi]
$$

is defined as a modified Legendre transform of $-\ln \mathcal{Z}_{k}[J]$ which includes the subtraction of $\Delta S_{k}[\phi]$. Here $\phi(\mathbf{r})=$ $\langle\varphi(\mathbf{r})\rangle$ is the order parameter (in the presence of the external source $J$ ). Provided that the initial value $k_{\text {in }}$ of $k$ is sufficiently large wrt the UV momentum cutoff $\Lambda$ of the sine-Gordon model, all fluctuations are completely frozen by $\Delta S_{k_{\text {in }}}[\varphi]$ and $\Gamma_{k_{\text {in }}}[\phi]=S[\phi]$. On the other hand the effective action of the sine-Gordon model is given by $\Gamma_{k=0}$ since $\Delta S_{k=0}=0$. The FRG approach aims at determining $\Gamma_{k=0}$ from $\Gamma_{\Lambda}$ using Wetterich's equation [4-6]

$$
\partial_{k} \Gamma_{k}[\phi]=\frac{1}{2} \operatorname{Tr}\left\{\partial_{k} R_{k}\left(\Gamma_{k}^{(2)}[\phi]+R_{k}\right)^{-1}\right\},
$$

where $\Gamma_{k}^{(2)}[\phi]$ denotes the second-order functional derivative of $\Gamma_{k}[\phi]$.

\section{DERIVATIVE EXPANSION}

To solve the exact flow equation (6) we use a derivative expansion of the scale-dependent effective action. Such an expansion is made possible by the regulator term $\Delta S_{k}$, which ensures that all vertices $\Gamma_{k}^{(n)}\left(\mathbf{q}_{1} \cdots \mathbf{q}_{n}\right)$ are smooth functions of momenta $\mathbf{q}_{i}$ and can be expanded in powers of $\mathbf{q}_{i}^{2} / \max (k, M)^{2}$ when $\left|\mathbf{q}_{i}\right| \ll \max (k, M)$ with $M$ the smallest mass in the spectrum. Thus the derivative expansion is sufficient to compute the soliton and breather masses in the massive phase as well as the expectation value of the exponential fields.

To second order of the derivative expansion, the effective action

$$
\Gamma_{k}[\phi]=\int d^{2} r\left\{\frac{1}{2} Z_{k}(\phi)(\nabla \phi)^{2}+U_{k}(\phi)\right\}
$$

is fully determined by two functions of the field, $Z_{k}(\phi)$ and $U_{k}(\phi)$, which are periodic on the interval ] $\pi / \beta, \pi / \beta]$. In practice we consider the dimensionless functions

$$
\tilde{U}_{k}(\phi)=\frac{U_{k}(\phi)}{Z_{k} k^{2}}, \quad \tilde{Z}_{k}(\phi)=\frac{Z_{k}(\phi)}{Z_{k}},
$$

where $Z_{k}$ is defined from the condition

$$
\frac{\beta}{2 \pi} \int_{-\pi / \beta}^{\pi / \beta} d \phi \tilde{Z}_{k}(\phi)=1
$$

The flow equations for the functions $\tilde{U}_{k}(\phi)$ and $\tilde{Z}_{k}(\phi)$, obtained by inserting (7) into (6), are given in Appendix .

\section{CHOICE OF THE REGULATOR FUNCTION $R_{k}$}

The initial value of the running momentum scale must satisfy $k_{\text {in }} \gg \Lambda$ in order to ensure that all fluctuations are frozen and $\Gamma_{k_{\text {in }}}[\phi]=S[\phi]$. In practice we take $k_{\text {in }} \sim$ $100 \Lambda$. Figure 5 shows the mass $M$ obtained from FRG with different regulator functions:

$$
r(y)=\left\{\begin{array}{l}
\frac{\alpha}{e^{y}-1} \\
\alpha \frac{(1-y)^{2}}{y} \Theta(1-y) \\
\alpha \frac{e^{-y}}{y}(1+\gamma y)
\end{array}\right.
$$

( $\alpha, \gamma$ are free parameters). In the range $K \leq 0.4$, the accuracy of the FRG result is better than $2 \%$ regardless of the choice of the regulator. For $K>0.4$ it deteriorates and the results become strongly dependent on the regulator. Note however that although the relative error can be larger than $100 \%$, the absolute error remains small since the mass $M$ goes to zero as $K \rightarrow 1$. 


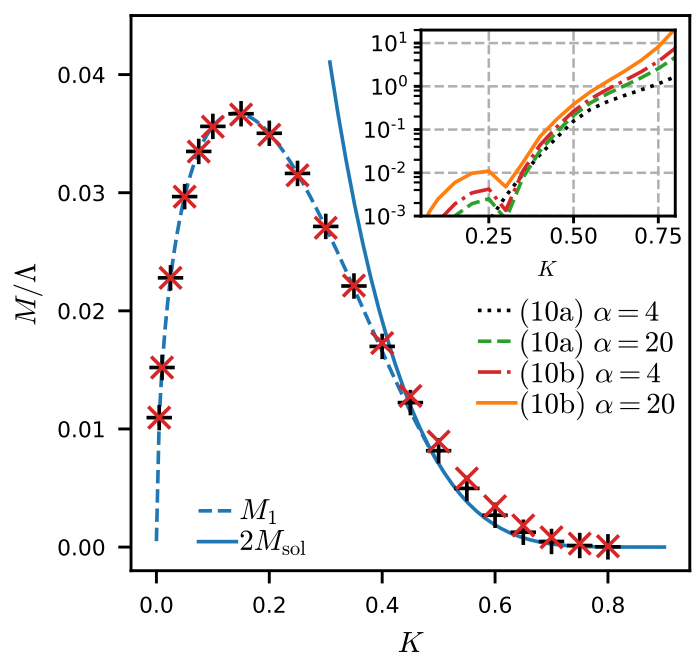

Figure 5. Mass $M$ as obtained from the FRG approach with the regulator functions (10a) $(+)$ and $(10 \mathrm{~b})(\times)$ and $\alpha=4$. The solid and dashed lines show the exact values of $2 M_{\text {sol }}$ and $M_{1}$ (the latter being defined only for $K \leq 1 / 2$ ). The inset shows the relative error of the FRG result for the same regulator functions and $\alpha=4$ or $\alpha=20$. The regulator function (10c) gives results (not shown) similar to (10a). (Here and in the following figures: $\Lambda=1$ and $u / \Lambda^{2}=10^{-3}$.)

\section{THE NONUNIVERSAL SCALE FACTOR $b$}

In a theory with a UV momentum cutoff $\Lambda$ the soliton mass is given by [7]

$$
M_{\mathrm{sol}}=\frac{2 \Gamma\left(\frac{K}{2-2 K}\right)}{\sqrt{\pi} \Gamma\left(\frac{1}{2-2 K}\right)}\left[\frac{\Gamma(1-K)}{\Gamma(K)} \frac{\pi}{2} u \Lambda_{\mathcal{R}}^{-2 K}\right]^{\frac{1}{2-2 K}} .
$$

The value of $\Lambda_{\mathcal{R}}$ (which depends on $\Lambda$ ) can be determined by considering the one-loop correction to the mass $M_{1, \mathrm{cl}}=\sqrt{8 \pi K u}$ of the lightest breather in the semiclassical limit $K \rightarrow 0$ [7], i.e.

$$
\frac{M_{1}^{2}}{M_{1, \mathrm{cl}}^{2}}=1+2 K \ln \left(\frac{M_{1, \mathrm{cl}} e^{C}}{2 \Lambda_{\mathcal{R}}}\right),
$$

where $C$ is the Euler constant. An elementary calculation based on the action (1) gives [1]

$$
\frac{M_{1}^{2}}{M_{1, \mathrm{cl}}^{2}}=1-2 K \int_{0}^{\infty} d q \frac{q}{q^{2}+M_{1, \mathrm{cl}}^{2}} f_{\Lambda}(q),
$$

where $f_{\Lambda}(q)$ is a momentum cutoff function (e.g. $f_{\Lambda}(q)=$ $\Theta(\Lambda-q)$ for a hard cutoff). By comparing (12) and (13), we find that $\Lambda$ and $\Lambda_{\mathcal{R}}$ are related by

$$
\ln \left(\frac{2 \Lambda_{\mathcal{R}} e^{-C}}{M_{1, \mathrm{cl}}}\right)=\int_{0}^{\infty} d q \frac{q}{q^{2}+M_{1, \mathrm{cl}}^{2}} f_{\Lambda}(q)
$$

For $M_{1, \mathrm{cl}} \ll \Lambda$, the rhs gives $\ln \left(\Lambda / M_{1, \mathrm{cl}}\right)+$ const and Eq. (14) allows us to determine the scale factor $b=\Lambda_{\mathcal{R}} / \Lambda$

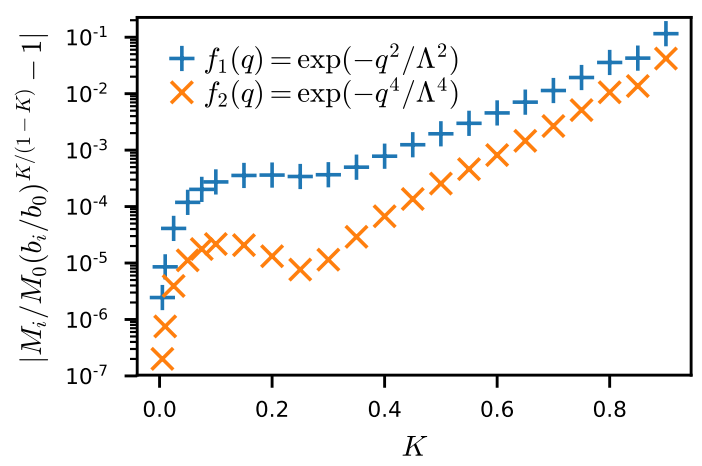

Figure 6. $\left|\left(M_{i} / M_{0}\right)\left(b_{i} / b_{0}\right)^{K /(1-K)}-1\right|$ for fixed values of $\Lambda$ and $u / \Lambda^{2}$ and two different cutoff functions, $f_{1}(q)$ and $f_{2}(q)$. $M_{0}$ is the mass obtained with the hard cutoff $f_{\Lambda}(q)=\Theta(\Lambda-$ $q)$. The scale parameter $b=\Lambda_{\mathcal{R}} / \Lambda$ is obtained from (14).

for a given cutoff function $f_{\Lambda}(q)$ (for a hard cutoff $b=$ $\left.e^{C} / 2\right)$. The soliton mass in the model defined with a momentum cutoff $\Lambda$ can therefore be written as

$$
M_{\mathrm{sol}}=b \Lambda \frac{2 \Gamma\left(\frac{K}{2-2 K}\right)}{\sqrt{\pi} \Gamma\left(\frac{1}{2-2 K}\right)}\left[\frac{\Gamma(1-K)}{\Gamma(K)} \frac{\pi u}{2(b \Lambda)^{2}}\right]^{\frac{1}{2-2 K}},
$$

where $b=\Lambda_{\mathcal{R}} / \Lambda$ is obtained from (14).

\section{UNIVERSALITY}

Equation (15) implies universality in the sense that $M_{\text {sol }} / \Lambda$ is a universal function of $K$ and $u / \Lambda^{2}$ up to the scale factor $b$. This universality is due to the flow being controlled by the Gaussian fixed point $u=0$ for $M_{\text {sol }} \ll$ $k \ll \Lambda$. In this momentum range all correlation functions exhibit the same scaling, e.g. $\left\langle e^{i \beta \varphi(\mathbf{r})} e^{-i \beta \varphi\left(\mathbf{r}^{\prime}\right)}\right\rangle \sim$ $\left(\left|\mathbf{r}-\mathbf{r}^{\prime}\right| \Lambda\right)^{-4 K}$, up to a nonuniversal prefactor that depends on the cutoff function $f_{\Lambda}(q)$. Various theories, corresponding to different implementations of the cutoff (or, equivalently, different normalizations of the correlation functions), are simply related by the scale factor $b$.

In Fig. 6 we show the ratio

$$
\frac{M_{i}}{M_{0}}\left(\frac{b_{i}}{b_{0}}\right)^{\frac{K}{1-K}}
$$

where $M_{0}$ is the mass obtained with the hard cutoff $f_{\Lambda}(q)=\Theta(\Lambda-q)$ and $M_{i}$ that obtained with the cutoff $f_{1}(q)=e^{-q^{2} / \Lambda^{2}}$ or $f_{2}(q)=e^{-q^{4} / \Lambda^{4}}$. The scale parameter $b=\Lambda_{\mathcal{R}} / \Lambda$ is obtained from (14). Universality implies that the ratio (16) is equal to one. This property is satisfied with a very high accuracy (the ratio differs from one by less than $10^{-3}$ ) in the range $K \leq 0.4$ where the mass $M$ is obtained with high precision. Not surprisingly, deviations from universality are more pronounced for $K>0.4$ due to the lesser accuracy of the FRG results. 


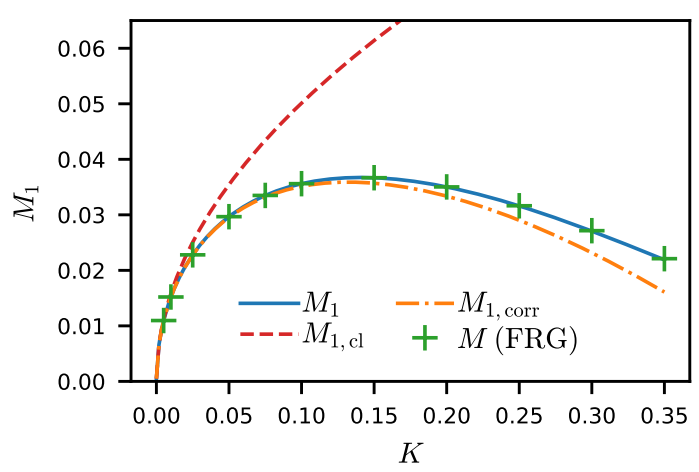

Figure 7. Mass $M_{1}$ of the lightest breather for $K \ll 1$. The FRG result is compared to the leading-order (semiclassical) value $M_{1, \mathrm{cl}}=\sqrt{8 \pi K u}$ and the next-to-leading-


$M_{1}=2 M_{\mathrm{sol}} \sin \left(\frac{\pi K}{2(1-K)}\right)$ given by $(15)$.

\section{SEMICLASSICAL LIMIT}

In the limit $K \ll 1$, the leading correction to the mass $M_{1, \mathrm{cl}}$ of the lightest breather is given by Eq. (12) with $\Lambda_{\mathcal{R}}=b \Lambda$. When $K \rightarrow 0$ the partition function can be computed by a saddle-point approximation and the initial value $\Gamma_{k_{\mathrm{in}}}[\phi]=S[\phi]$ of the effective action gives the exact result $M_{1, \mathrm{cl}}=\sqrt{8 \pi K u}$ (the flow does not bring any correction to $\Gamma_{k_{\text {in }}}[\phi]=S[\phi]$ when $K \rightarrow 0$ ). In Fig. 7 we compare the FRG result for $M_{1}$ with the exact value, the semiclassical limit $K \rightarrow 0$ and the perturbative result (12). For $K \lesssim 0.1$, the agreement between FRG and the exact solution is nearly perfect.

\section{EXPECTATION VALUE OF EXPONENTIAL FIELDS}

To compute the expectation value of the exponential fields, we add to the action the source term

$$
S_{h}[\varphi]=-\int d^{2} r\left(h^{*} e^{i n \sqrt{2 \pi K} \varphi}+\text { c.c. }\right)
$$

so that $\left\langle e^{i n \sqrt{2 \pi K} \varphi(\mathbf{r})}\right\rangle$ can be obtained from $\ln \mathcal{Z}_{k}\left[J, h^{*}, h\right]$ by functional differentiation wrt $h^{*}(\mathbf{r})$. The scaledependent effective action reads

$$
\Gamma_{k}\left[\phi, h^{*}, h\right]=-\ln \mathcal{Z}_{k}\left[J, h^{*}, h\right]+\int d^{2} r J \phi-\Delta S_{k}[\phi]
$$

and satisfies the flow equation

$$
\partial_{t} \Gamma_{k}\left[\phi, h^{*}, h\right]=\frac{1}{2} \operatorname{Tr}\left\{\partial_{t} R_{k}\left(\Gamma^{(2)}\left[\phi, h^{*}, h\right]+R_{k}\right)^{-1}\right\}
$$

with initial conditions $\Gamma_{k_{\mathrm{in}}}\left[\phi, h^{*}, h\right]=S[\phi]+S_{h}[\phi]$.

We consider the ansatz

$$
\Gamma_{k}\left[\phi, h^{*}, h\right]=\int d^{2} r\left\{\frac{1}{2} Z_{k}\left(\phi, h^{*}, h\right)(\nabla \phi)^{2}\right.
$$
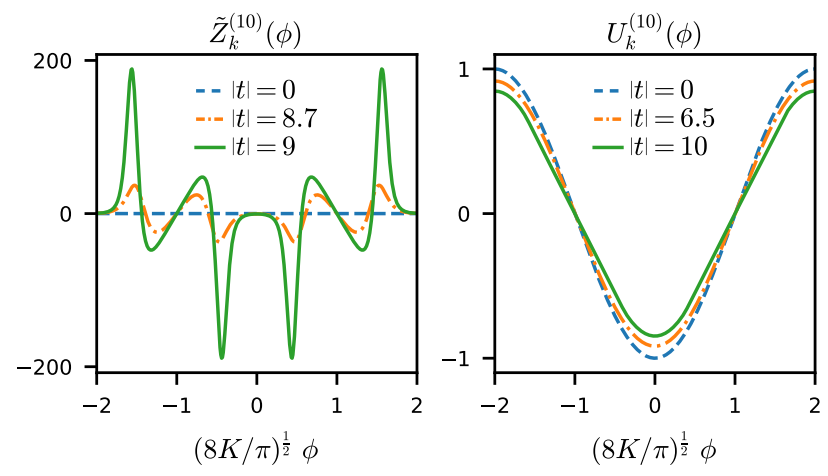

Figure 8. $\quad \tilde{Z}_{k}^{(10)}(\phi)$ and $U_{k}^{(10)}(\phi)$ for various values of $t=$ $\ln \left(k / k_{\text {in }}\right)$ and $n=1$.

$$
\left.+U_{k}\left(\phi, h^{*}, h\right)\right\} \text {. }
$$

The expectation value of interest reads

$$
\left\langle e^{i n \sqrt{2 \pi K} \varphi(\mathbf{r})}\right\rangle=-\left.\frac{\partial U_{k}\left(\phi, h^{*}, h\right)}{\partial h^{*}}\right|_{\substack{h^{*}=h=0 \\ \phi=0}} \equiv-U_{k}^{(10)}(\phi=0),
$$

noting that $\phi=0$ corresponds to the minimum of the potential $U\left(\phi, h^{*}, h\right)$ when $h^{*}=h=0$. The RG equation for $U_{k}^{(10)}(\phi)$ involves $\left.Z_{k}^{(10)}(\phi) \equiv \partial_{h^{*}} Z_{k}\left(\phi, h^{*}, h\right)\right|_{h^{*}=h=0}$. In practice we consider the dimensionless functions

$$
\tilde{U}_{k}^{(10)}(\phi)=\frac{U_{k}^{(10)}(\phi)}{Z_{k} k^{2}}, \quad \tilde{Z}_{k}^{(10)}(\phi)=\frac{Z_{k}^{(10)}(\phi)}{Z_{k}} .
$$

The flow equations satisfied by $\tilde{U}_{k}^{(10)}(\phi)$ and $\tilde{Z}_{k}^{(10)}(\phi)$ are given in Appendix . The results obtained in the massive phase are shown in Fig. 8 for $n=1$. 


\section{APPENDIX A: FLOW EQUATIONS}

The flow equations read

$$
\begin{aligned}
& \partial_{t} \tilde{U}_{k}=\left(\eta_{k}-2\right) \tilde{U}_{k}+\frac{1}{4 \pi Z_{k}} l_{0,0}^{2} \\
& \partial_{t} \tilde{Z}_{k}=\eta_{k} \tilde{Z}_{k}-\frac{1}{4 \pi Z_{k}}\left[2 \tilde{U}_{k}^{\prime \prime \prime} l_{0,2}^{6} \tilde{Z}_{k}^{\prime}-2 \tilde{U}_{k}^{\prime \prime \prime} l_{2,0}^{2} \tilde{Z}_{k}^{\prime}+\tilde{U}_{k}^{\prime \prime \prime 2} l_{0,2}^{4}+l_{1,0}^{2} \tilde{Z}_{k}^{\prime \prime}+l_{0,2}^{8} \tilde{Z}_{k}^{\prime 2}-\frac{5}{2} l_{2,0}^{4} \tilde{Z}_{k}^{\prime 2}\right]
\end{aligned}
$$

and

$$
\begin{aligned}
\partial_{t} \tilde{U}_{k}^{(10)}= & \left(\eta_{k}-2\right) \tilde{U}_{k}^{(10)}-\frac{1}{4 \pi Z_{k}}\left[l_{1,0}^{2} \tilde{U}_{k}^{(10) \prime \prime}+l_{1,0}^{4} \tilde{Z}_{k}^{(10)}\right], \\
\partial_{t} \tilde{Z}_{k}^{(10)}= & \eta_{k} \tilde{Z}_{k}^{(10)}-\frac{1}{4 \pi Z_{k}}\left\{2 l_{0,2}^{6}\left[\tilde{U}_{k}^{(10) \prime \prime \prime} \tilde{Z}_{k}^{\prime}+\tilde{U}_{k}^{\prime \prime \prime} \tilde{Z}_{k}^{(10) \prime}\right]+2 \tilde{U}_{k}^{(10) \prime \prime \prime} \tilde{U}_{k}^{\prime \prime \prime} l_{0,2}^{4}-8 \tilde{U}_{k}^{\prime \prime \prime} l_{1,2}^{6} \tilde{U}_{k}^{(10) \prime \prime} \tilde{Z}_{k}^{\prime}\right. \\
& +4 \tilde{U}_{k}^{\prime \prime \prime} l_{3,0}^{2} \tilde{U}_{k}^{(10) \prime \prime} \tilde{Z}_{k}^{\prime}-4 \tilde{U}_{k}^{\prime \prime \prime 2} l_{1,2}^{4} \tilde{U}_{k}^{(10) \prime \prime}-4 l_{1,2}^{8} \tilde{U}_{k}^{(10) \prime \prime} \tilde{Z}_{k}^{\prime 2}+5 l_{3,0}^{4} \tilde{U}_{k}^{(10) \prime \prime} \tilde{Z}_{k}^{\prime 2}-l_{2,0}^{2}\left[2 \tilde{U}_{k}^{(10) \prime \prime \prime} \tilde{Z}_{k}^{\prime}\right. \\
& \left.+\tilde{U}_{k}^{(10) \prime \prime} \tilde{Z}_{k}^{\prime \prime}+2 \tilde{U}_{k}^{\prime \prime \prime} \tilde{Z}_{k}^{(10) \prime}\right]-8 \tilde{U}_{k}^{\prime \prime \prime} \tilde{Z}_{k}^{(10)} l_{1,2}^{8} \tilde{Z}_{k}^{\prime}+2 \tilde{U}_{k}^{\prime \prime \prime} \tilde{Z}_{k}^{(10)} l_{2,1}^{6} \tilde{Z}_{k}^{\prime}+8 \tilde{U}_{k}^{\prime \prime \prime} \tilde{Z}_{k}^{(10)} l_{3,0}^{4} \tilde{Z}_{k}^{\prime}-4 \tilde{U}_{k}^{\prime \prime \prime} \tilde{Z}_{k}^{(10)} l_{1,2}^{6} \\
& +\tilde{U}_{k}^{\prime \prime \prime} \tilde{Z}_{k}^{(10)} l_{2,1}^{4}+\tilde{U}_{k}^{\prime \prime \prime} \tilde{Z}_{k}^{(10)} l_{3,0}^{2}+l_{1,0}^{2} \tilde{Z}_{k}^{(10) \prime \prime}+2 l_{0,2}^{8} \tilde{Z}_{k}^{(10) \prime} \tilde{Z}_{k}^{\prime}-l_{2,0}^{4}\left[5 \tilde{Z}_{k}^{(10) \prime} \tilde{Z}_{k}^{\prime}+\tilde{Z}_{k}^{(10)} \tilde{Z}_{k}^{\prime \prime}\right] \\
& \left.-4 \tilde{Z}_{k}^{(10)} l_{1,2}^{10} \tilde{Z}_{k}^{\prime 2}+\tilde{Z}_{k}^{(10)} l_{2,1}^{8} \tilde{Z}_{k}^{\prime 2}+8 \tilde{Z}_{k}^{(10)} l_{3,0}^{6} \tilde{Z}_{k}^{\prime 2}\right\},
\end{aligned}
$$

where $t=\ln \left(k / k_{\text {in }}\right)$ and $\eta_{k}=-\partial_{t} \ln Z_{k}$. The equation for $\eta_{k}$ is simply derived from (9). To alleviate the notations we do not write explicitly the $\phi$ dependence of $\tilde{U}_{k}(\phi), \tilde{Z}_{k}(\phi), \tilde{U}_{k}^{(10)}(\phi)$ and $\tilde{Z}_{k}^{(10)}(\phi)$. The initial conditions are

$$
\tilde{U}_{k_{\mathrm{in}}}(\phi)=-\left(u / k_{\mathrm{in}}^{2}\right) \cos (\beta \phi), \quad \tilde{Z}_{k_{\mathrm{in}}}(\phi)=1, \quad \tilde{U}_{k_{\mathrm{in}}}^{(10)}(\phi)=-e^{i n \sqrt{2 \pi K} \phi} / k_{\mathrm{in}}^{2}, \quad \tilde{Z}_{k_{\mathrm{in}}}^{(10)}(\phi)=0
$$

and $Z_{k_{\mathrm{in}}}=1$. The threshold function $l_{n, m}^{d}$ is defined in Appendix .

\section{Weak-coupling limit}

The perturbative flow equations are recovered by retaining a single harmonic of the potential, i.e. $\tilde{U}_{k}(\phi)=$ $-\tilde{u}_{k} \cos (\beta \phi)$, and neglecting the flow of $\tilde{Z}_{k}(\phi)$. Using

$$
l_{0,0}^{2}\left(\tilde{U}^{\prime \prime}, 1,0\right)=l_{0,0}^{2}(0,1,0)-l_{1,0}^{2}(0,1,0) \tilde{U}^{\prime \prime}+\mathcal{O}\left(\tilde{U}^{\prime \prime 2}\right),
$$

where $l_{1,0}^{2}(0,1,0)=1$ is universal (i.e. independent of the regulator function $r$ ), one obtains

$$
\begin{aligned}
\partial_{t} \tilde{u}_{k} & =-2 \tilde{u}_{k}\left(1-K_{k}\right)+\mathcal{O}\left(\tilde{u}_{k}^{2}\right), \\
\partial_{t} K_{k} & =\tilde{u}_{k}^{2}\left(8 \pi K^{2}\right)^{2} l_{0,2}^{4}(0,1,0)+\mathcal{O}\left(\tilde{u}_{k}^{3}\right),
\end{aligned}
$$

where $K_{k}=K / Z_{k}$ is a "running" Luttinger parameter. Sufficiently far away from the BKT point $\left(K_{k}=1, \tilde{u}_{k}=0\right)$, one can neglect the renormalization of $K_{k}$ when $\tilde{u}_{k} \rightarrow 0$ and the flow equations become $\partial_{t} \tilde{u}_{k}=-2 \tilde{u}_{k}(1-K)$ to leading order. For $K<1, \tilde{u}_{k}$ grows as $k^{2 K-2}$. This is the massive phase studied in the Letter. When $1-K_{k}$ and $\tilde{u}_{k}$ are of the same order, both equations in (29) must be solved simultaneously and one recovers the scaling behavior of the BKT phase transition [9].

\section{APPENDIX B: THRESHOLD FUNCTIONS}

The threshold function $l_{n_{1}, n_{2}}^{d} \equiv l_{n_{1}, n_{2}}^{d}\left(\tilde{U}_{k}^{\prime \prime}, \tilde{Z}_{k}, \eta_{k}\right)$ is defined by

$$
\begin{aligned}
l_{0,0}^{d} & =\int_{0}^{\infty} d \tilde{q} \tilde{q}^{d-1} \frac{\dot{R}_{k}}{Z_{k} k^{2}} \tilde{G}, \\
l_{n_{1}, n_{2}}^{d} & =-\tilde{\partial}_{t} \int_{0}^{\infty} d \tilde{q} \tilde{q}^{d-1} \tilde{G}^{n_{1}} \tilde{G}^{\prime n_{2}} \quad \text { if } \quad\left(n_{1}, n_{2}\right) \neq(0,0),
\end{aligned}
$$


where

$$
\begin{aligned}
& \tilde{G}=\left[\left(\tilde{Z}_{k}+r\right) y+\tilde{U}_{k}^{\prime \prime}\right]^{-1}, \quad \tilde{\partial}_{t} \tilde{G}=-\frac{\dot{R}_{k}}{Z_{k} k^{2}} \tilde{G}^{2} \\
& \tilde{G}^{\prime}=-\tilde{G}^{2}\left[\tilde{Z}_{k}+r+y r^{\prime}\right], \quad \tilde{\partial}_{t} G^{\prime}=2 \frac{\dot{R}_{k}}{Z_{k} k^{2}} \tilde{G}^{3}\left[\tilde{Z}_{k}+r+y r^{\prime}\right]-\frac{\dot{R}_{k}^{\prime}}{Z_{k}} \tilde{G}^{2},
\end{aligned}
$$

and

$$
\begin{aligned}
& R_{k}=Z_{k} k^{2} y r, \quad \dot{R}_{k}=-Z_{k} k^{2} y\left(\eta_{k} r+2 y r^{\prime}\right), \\
& R_{k}^{\prime}=Z_{k}\left(r+y r^{\prime}\right), \quad \dot{R}_{k}^{\prime}=-Z_{k}\left[\eta_{k}\left(r+y r^{\prime}\right)+2 y\left(2 r^{\prime}+y r^{\prime \prime}\right)\right],
\end{aligned}
$$

with $y=\tilde{q}^{2}=\mathbf{q}^{2} / k^{2}, r \equiv r(y), r^{\prime} \equiv r^{\prime}(y)$, etc. The prime denotes a derivative wrt $y$, the dot a derivative wrt $t$ and $\tilde{\partial}_{t}$ acts only on the $k$ dependence of $R_{k}$.

[1] J. Berges, N. Tetradis, and C. Wetterich, "Nonperturbative renormalization flow in quantum field theory and statistical physics," Phys. Rep. 363, 223 (2002).

[2] B. Delamotte, "An Introduction to the Nonperturbative Renormalization Group," in Renormalization Group and Effective Field Theory Approaches to Many-Body Systems, Lecture Notes in Physics, Vol. 852, edited by A. Schwenk and J. Polonyi (Springer Berlin Heidelberg, 2012) pp. 49132.

[3] P. Kopietz, L. Bartosch, and F. Schütz, Introduction to the Functional Renormalization Group (Springer, Berlin, 2010).
[4] C. Wetterich, "Exact evolution equation for the effective potential," Phys. Lett. B 301, 90 (1993).

[5] Ulrich Ellwanger, "Flow equations for $n$ point functions and bound states," Z. Phys. C 62, 503-510 (1994).

[6] T. R. Morris, "The exact renormalization group and approximate solutions," Int. J. Mod. Phys. A 09, 2411-2449 (1994).

[7] Al. B. Zamolodchikov, "Mass scale in the sine-Gordon model and its reductions," Int. J. Mod. Phys. A 10, 11251150 (1995).

[8] R. Rajaraman, Solitons and instantons (North-Holland, Amsterdam, 1989).

[9] See, e.g., P. M. Chaikin and T. C. Lubensky, Principles of Condensed Matter Physics (Cambridge University Press, 1995). 